(3)

\title{
APPLICATIONS OF THE GREEN FUNCTIONS IN THE STUDY OF ACOUSTIC PROBLEMS IN OPEN AND CLOSED SPACES
}

\author{
A. Tadeu, J. António and L. Godinho \\ Department of Civil Engineering Faculty of Sciences and Technology, University of Coimbra, \\ P-3030-290 Coimbra, Portugal.E-mail:tadeu@dec.uc.pt
}

(Received 11 July 2000, and in final form 9 March 2001)

\begin{abstract}
This paper presents a set of analytical solutions (Green functions) for the steady state response of a homogenous acoustic three-dimensional space subjected to a point harmonic load or a spatially sinusoidal harmonic line load. The propagation medium is modelled with plane surfaces placed so as to reproduce spaces that vary from a simple half-space to a rectangular parallelepiped closed space. The final expressions are implemented to evaluate first the pressure field inside a rectangular parallelepiped room, whose walls allow different absorption coefficients. Then, the acoustic scattering of a three-dimensional sound source by an infinitely long rigid barrier in the vicinity of a tall building is evaluated using the boundary-element method (BEM), making use of the analytical solution for a spatially sinusoidal line source. The use of these Green functions allows the required BEM discretization to be limited to the surface of the barrier, avoiding the discretization of the tall building and ground. The calculations are performed in the frequency domain and time signatures are obtained by means of inverse Fourier transforms. Complex frequencies are used to attenuate the response at the end of the time frame, which is taken into account by rescaling the time response.
\end{abstract}

(C) 2001 Academic Press

\section{INTRODUCTION}

The propagation of acoustic waves in the presence of open or closed spaces, such as in the vicinity of acoustic barriers or inside parallelepiped closed rooms, involves frequency values that tend to rule out the use of many numerical techniques, because of the computer effort required.

Sound propagation in closed spaces is often analyzed by means of the ray-tracing and image model techniques [1-4]. The ray-tracing technique follows only a limited number of acoustic rays between the source and the receiver points. This procedure allows the resulting simulation to be calculated in a reasonable time, even when more complicated geometries are studied. However, the number of rays needed to corroborate the correctness of the numerical calculation remains uncertain. Furthermore, certain rays that would contribute to the correct results might not be included in the analysis [5]. In the image model technique, the acoustic field is calculated by adding the contribution of virtual sources (image sources) to it, placed so as to simulate the direct and successive multi-reflections produced by the reflecting walls. Unlike the ray-tracing technique, there is no uncertainty involved, but the analysis of models with irregular geometry is complex, and calculations may result in high computational cost when higher order reflections are taken into consideration. 
Diffraction-based methods have been used to compute the acoustic energy loss caused by the insertion of acoustic barriers placed in open spaces [6,7]. Other numerical techniques, such as the boundary-element method (BEM) or the finite-element method, may model the problem more realistically. However, these methods are costly in terms of computational effort, and are therefore difficult to apply to very high frequencies. The application of these numerical methods has mostly been restricted to situations where the solution is required within two-dimensional domains. The evaluation of the full scattering wave field generated by sources placed in the presence of three-dimensional propagation media requires the use of computationally demanding numerical schemes.

Lacerda et al. [8] proposed a dual boundary-element formulation for analyzing two-dimensional sound propagation in the vicinity of acoustic barriers, over an infinite plane, in which both the ground and the barrier were assumed to be absorptive. The three-dimensional propagation of sound around an absorptive finite barrier has been studied by Lacerda et al. [9], where a dual boundary-element formulation that allowed the barrier to be modelled as a simple surface was introduced.

The solution becomes much simpler if the medium is two-dimensional (2-D), even if the dynamic source is three-dimensional (3-D), such as a point load. Such a situation is frequently referred to as a two-and-a-half-dimensional problem (or 2-1/2-D for short), for which solutions can be obtained by means of a spatial Fourier transform in the direction in which the geometry does not vary. For this, a sequence of 2-D problems must be solved, with different spatial wavenumbers, $k_{z}$, associated with different spatially sinusoidal harmonic line loads.

Zhang and Chopra [10] used a BEM formulation to compute the 3-D seismic response of an infinitely long canyon with an arbitrary cross-section in a homogeneous visco-elastic half-space. The dynamic excitation was assumed to be represented by Rayleigh surface waves or plane body waves arriving at the half-space from any direction. The 3-D boundary integral equation is reduced to a set of 2-D problems by Fourier transforming the 3-D Green functions along the canyon axis. Stamos and Beskos [11] used a direct BEM to describe the 3-D dynamic response of long-lined tunnels with a uniform cross-section, buried in an elastic or visco-elastic half-space, and subjected to plane harmonic waves propagating in arbitrary directions. The problem is again reduced to a 2-D one by a co-ordinate transformation and appropriate integration of the full space dynamic fundamental solution along the direction of the tunnel axis.

Our work first uses the image model technique to define analytical solutions for the propagation of sound in 3- and 2-D spaces in the presence of point and linear sound sources. This work extends the work of Allen and Berkley [1], who computed the response between two points in a small rectangular room using the image technique. Their model is built on the impulse response in the time domain, which can be later convolved with any type of input signal. Thus, the computed impulse response is built up as a "histogram" of image sources received at different time delays. In our method, the problem is solved in the frequency domain, and the time responses are obtained by means of inverse Fourier transforms. Furthermore, the Green functions are presented for different geometries.

Our model is then extended to handle a situation where linear harmonic sources, varying sinusoidally in the third dimension, are excited. These solutions (Green functions) can be of significant value in the formulation of 2-D and 3-D elastodynamic problems, via boundary elements, together with integral transforms [10-13].

To illustrate the importance of these equations, the first set of equations is implemented and used to calculate the field generated by a point pressure load inside a rectangular parallelepiped closed room (whose opposite faces are parallel and the adjoining faces are perpendicular), while the second set is used to compute the acoustic scattering of a 3-D 
sound pressure source by an infinitely long rigid barrier in the vicinity of a tall building. The model defines the BEM equations by discretizing the barrier alone, while the appropriate Green functions, which are defined in this paper, accomodate the far field radiation conditions and the boundary conditions on the floor and building. This example extends the work of Jean et al. [14], where frequency-domain responses are calculated for an isolated barrier and the importance of the source type for insertion loss is assessed.

The solutions are first computed in the frequency domain. The time signatures are then calculated by using inverse Fourier transforms. Complex frequencies $\left(\omega_{c}=\omega-i \eta\right)$ are used to determine the acoustic signals, making it possible to attenuate the response at the end of the time frame. The effect of the complex part of the frequency is taken into account in the time domain, rescaling the response by applying an exponential function $\mathrm{e}^{\eta t}[15]$.

\section{INCIDENT FIELD GENERATED BY A POINT OR A LINEAR SOURCE WITH SPATIAL VARIATION IN $z$}

Consider first an infinite, homogeneous space, subjected to a harmonic point pressure load in the form $\delta\left(x-x_{0}\right) \delta\left(y-y_{0}\right) \delta\left(z-z_{0}\right) \mathrm{e}^{\mathrm{i} \omega t}$, acting in all directions at $\left(x_{0}, y_{0}, z_{0}\right)$. In this expression, $\delta(x), \delta(y)$ and $\delta(z)$ are Dirac delta functions and $\omega$ is the frequency of the load. The resulting pressure field generated by this acoustic load can be expressed by the equation

$$
p(\omega, x, y, z, t)=\frac{A \mathrm{e}^{\mathrm{i}(\omega / \alpha)\left(\alpha t-\sqrt{\left(x-x_{0}\right)^{2}+\left(y-y_{0}\right)^{2}+\left(z-z_{0}\right)^{2}}\right)}}{\sqrt{\left(x-x_{0}\right)^{2}+\left(y-y_{0}\right)^{2}+\left(z-z_{0}\right)^{2}}}
$$

in which $A$ is the wave amplitude, $\alpha$ the propagation velocity, and $\mathrm{i}=\sqrt{-1}$.

Consider next an infinite homogenous space subjected to a spatially varying line load of the form $\delta\left(x-x_{0}\right) \delta\left(y-y_{0}\right) \mathrm{e}^{\mathrm{i}\left(\omega t-k_{z}\left(z-z_{0}\right)\right)}$, with $k_{z}$ being the wavenumber along the $z$ direction. This load acts on point $\left(x_{0}, y_{0}\right)$ and varies sinusoidally in the direction $z$ (see Figure 2).

The response to this load can be obtained by Fourier-transforming equation (1) in the $z$ direction, and using the effective wavenumbers, $k_{\alpha}=\sqrt{\omega^{2} / \alpha^{2}-k_{z}^{2}}$ with $\operatorname{Im} k_{\alpha}<0$, to obtain

$$
\hat{p}\left(\omega, x, y, k_{z}\right)=B \mathrm{H}_{0}\left(k_{\alpha} \sqrt{\left(x-x_{0}\right)^{2}+\left(y-y_{0}\right)^{2}}\right),
$$

in which $\mathrm{H}_{0}(\ldots)$ are Hankel second functions of the order 0 , and $B=-\mathrm{i} A / 2$.

If one assumes the existence of an infinite set of evenly spaced sources along the $z$ direction, the former incident field may be written as

$$
p(\omega, x, y, z)=\frac{2 \pi}{L} \sum_{m=-\infty}^{m=\infty} \hat{p}\left(\omega, x, y, k_{z m}\right) \mathrm{e}^{\mathrm{i} k_{z m}\left(z-z_{0}\right)},
$$

where $L$ is the spatial source interval, and $k_{z m}=(2 \pi / L) m$. Thus, the 3 -D wave field may be obtained as the wave irradiated by a sum of harmonic (steady state) line loads whose amplitudes vary sinusoidally in the third dimension. This sum converges and can be approximated by a finite number of terms. Therefore, the 3-D solution can be obtained by superposing 2-D solutions characterized by specific axial wavelength values, $k_{z}$. Notice that $k_{z}=0$ corresponds to a constant linear source along the $z$ direction, i.e., the 2-D problem. 


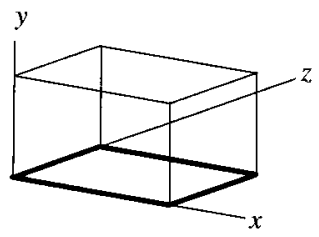

(a)

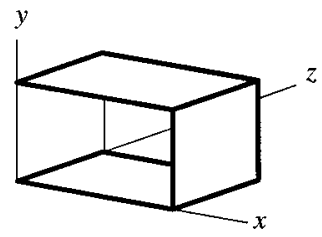

(d)

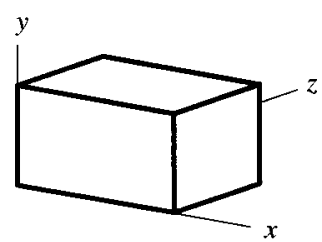

(g)

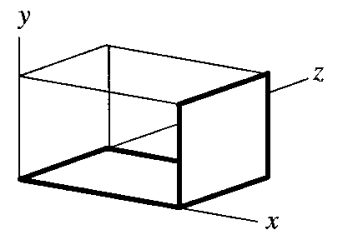

(b)

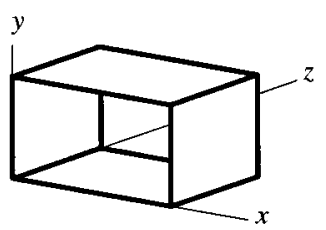

(e)

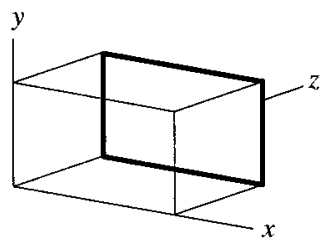

(h)

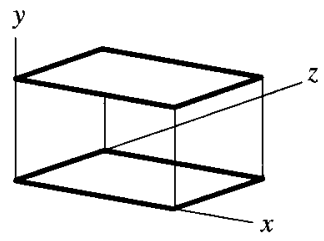

(c)

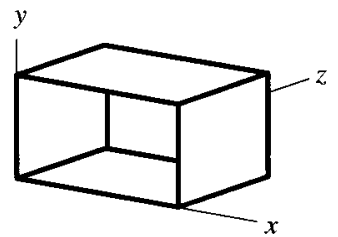

(f)

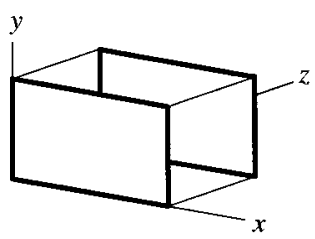

(i)

Figure 1. Models studied: (1) Geometry 1; (b) Geometry 2; (c) Geometry 3; (d) Geometry 4; (e) Geometry 5; (f) Geometry 6; (g) Geometry 7; (h) Geometry 8; (i) Geometry 9.

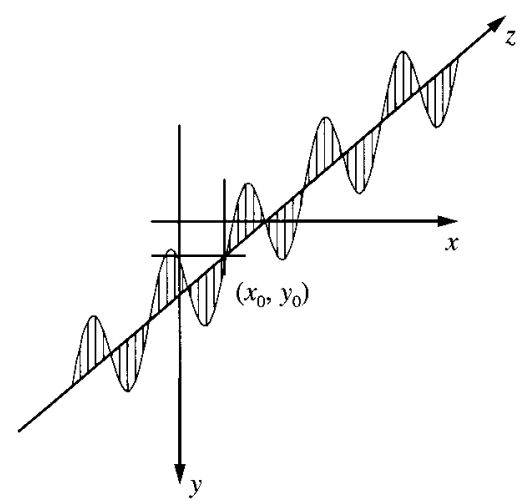

Figure 2. Linear source with spatial variation in $z$.

As described above, the Fourier transforms are arrived at, in mathematical terms, by adding together the periodic sources in the space $L=2 \pi / \Delta k_{z}$ (along the $z$-axis) and in time $T=2 \pi / \Delta \omega$, where $\Delta k_{z}$ is the interval of the wavelength and $\Delta \omega$ the frequency increment [16]. It is essential that the distance in space, $L$, is large enough to prevent the response being contaminated by the periodic sources, which means that the fictitious sources' contribution to the response, for times less than $T$, has to be nil. Complex frequencies, expressed by $\omega_{c}=\omega-$ i $\eta$ (with $\eta=0.7 \Delta \omega$ ), can be used to achieve this, since they permit 
the periodic sources to be reduced almost to the point where they are eradicated. An exponential function, $\mathrm{e}^{\eta t}$, is used in the time domain to offset the damping effect that results from applying this technique [15].

\section{3-D AND 2-1/2-D GREEN FUNCTIONS FOR ACOUSTIC PROBLEMS IN OPEN AND CLOSED SPACES}

The acoustic pressure field in the presence of perfectly reflecting barriers can be obtained by summing the effect of virtual image sources, placed in such a way as to verify the required boundary conditions, i.e., null pressure flows on the surface of the barriers. This gives a regular distribution of image sources, representing the first order images of the original source generated from the first reflections from the various walls and any other higher order image sources generated from the successive reflections from the corresponding combinations of reflecting walls. The number of image sources used is that which enables all the signals needed to determine the response in the time interval fixed by the frequency increment to be considered. Next, a complete set of Green functions is listed, giving the solution for different scenarios (as in Figure 1). For these different scenarios, the number of barriers is increased to allow simulation of the pressure field propagation generated from a simple half-space to a rectangular parallelepiped closed space, with dimensions $d_{1}, d_{2}$ and $d_{3}$, along the $x, y$ and $z$ directions respectively.

\subsection{GREEN FUNCTIONS FOR INCIDENT POINT PRESSURE SOURCES}

(a) Horizontal barrier in position $y=0$ (see Figure 1(a)):

$$
p(\omega, x, y, z)=A \sum_{j=0}^{1} \frac{\mathrm{e}^{-\mathrm{i}(\omega / \alpha) r_{i j k}}}{r_{i j k}} \quad(i=0, k=0, n=0)
$$

with $r_{i j k}=\sqrt{\left(\underline{x}_{i}\right)^{2}+\left(y_{j}\right)^{2}+\left(\underline{z}_{k}\right)^{2}}$ defined as in Appendix A.

(b) Corner created by a vertical barrier located at $x=d_{1}$ and a horizontal one at $y=0$ (see Figure 1(b)):

$$
p(\omega, x, y, z)=A \sum_{j=0}^{1} \sum_{j=0}^{1} \frac{\mathrm{e}^{-\mathrm{i}(\omega / \alpha) r_{i j k}}}{r_{i j k}} \quad(k=0, n=0, m=-1) .
$$

(c) Layer bounded by horizontal barriers at distance $y=0$ and $d_{2}$ (see Figure 1(c)):

$$
p(\omega, x, y, z)=A\left\{\frac{\mathrm{e}^{-\mathrm{i}(\omega / \alpha) r_{000}}}{r_{000}}+\sum_{n=0}^{N S V}\left[\sum_{j=1}^{4} \frac{\mathrm{e}^{-\mathrm{i}(\omega / \alpha) r_{i j k}}}{r_{i j k}}\right]\right\} \quad(i=0, k=0) .
$$

The number of sources used (NSV) is determined such that all the signals needed to define the response in the time interval fixed by the frequency increment $(T=1 / \Delta f)$ are taken into consideration.

(d) Layer bounded by horizontal barriers at distance $y=0$ and $d_{2}$ and another, vertical, at $x=d_{1}$ (see Figure $\left.1(\mathrm{~d})\right)$ :

$$
p(\omega, x, y, z)=A \sum_{i=0}^{1}\left\{\frac{\mathrm{e}^{-\mathrm{i}(\omega / \alpha) r_{i 00}}}{r_{i 00}}+\sum_{n=0}^{N S V}\left[\sum_{j=1}^{4} \frac{\mathrm{e}^{-\mathrm{i}(\omega / \alpha) r_{i j k}}}{r_{i j k}}\right]\right\} \quad(k=0, m=-1) .
$$


(e) Tunnel created by horizontal barriers at distance $y=0$ and $d_{2}$ and vertical ones at $x=0$ and $d_{1}$ (see Figure 1(e)):

$$
\begin{aligned}
p(\omega, x, y, z)= & A\left\{\frac{\mathrm{e}^{-\mathrm{i}(\omega / \alpha) r_{000}}}{r_{000}}+\sum_{n=0}^{N S V}\left[\sum_{j=1}^{4} \frac{\mathrm{e}^{-\mathrm{i}(\omega / \alpha) r_{0 j k}}}{r_{0 j k}}\right]\right. \\
& \left.+\sum_{m=1}^{N S H}\left\{\sum_{i=0}^{4}\left\{\frac{\mathrm{e}^{-\mathrm{i}(\omega / \alpha) r_{i 00}}}{r_{i 00}}+\sum_{n=0}^{N S V}\left[\sum_{j=1}^{4} \frac{\mathrm{e}^{-\mathrm{i}(\omega / \alpha) r_{i j k}}}{r_{i j k}}\right]\right\}\right\}\right\} \quad(k=0),
\end{aligned}
$$

where NSH represents the number of sources used in the horizontal directions that are required to enable all the signals needed to define the response in the time interval fixed by the frequency increment to the considered.

(f) Tunnel created by horizontal barriers at distance $y=0$ and $d_{2}$ and vertical ones at $x=0$ and $d_{1}$, and bounded at $z$ by a barrier placed at $z=d_{3}$ (see Figure 1(f)):

$$
\begin{aligned}
p(\omega, x, y, z)= & A \sum_{k=0}^{1}\left\{\frac{\mathrm{e}^{-\mathrm{i}(\omega / \alpha) r_{00 k}}}{r_{00 k}}+\sum_{n=0}^{N S V}\left[\sum_{j=1}^{4} \frac{\mathrm{e}^{-\mathrm{i}(\omega / \alpha) r_{0 j k}}}{r_{0 j k}}\right]\right. \\
& \left.+\sum_{m=1}^{N S H}\left\{\sum_{i=0}^{4}\left\{\frac{\mathrm{e}^{-\mathrm{i}(\omega / \alpha) r_{i 00}}}{r_{i 00}}+\sum_{n=0}^{N S V}\left[\sum_{j=1}^{4} \frac{\mathrm{e}^{-\mathrm{i}(\omega / \alpha) r_{i j k}}}{r_{i j k}}\right]\right\}\right\}\right\} \quad(l=-1) .
\end{aligned}
$$

(g) Closed space bounded by horizontal barriers placed at $y=0$ and $d_{2}$ and vertical ones at $x=0, x=d_{1}, z=0$ and $d_{3}$ (see Figure $1(\mathrm{~g})$ ):

$$
\begin{aligned}
p(\omega, x, y, z)= & A\left\{\frac{\mathrm{e}^{-\mathrm{i}(\omega / \alpha) r_{000}}}{r_{000}}+\sum_{n=0}^{N S V}\left[\sum_{j=1}^{4} \frac{\mathrm{e}^{-\mathrm{i}(\omega / \alpha) r_{0 j 0}}}{r_{0 j 0}}\right]\right. \\
& \left.+\sum_{m=1}^{N S H} \sum_{i=1}^{4}\left\{\frac{\mathrm{e}^{-\mathrm{i}(\omega / \alpha) r_{i 00}}}{r_{i 00}}+\sum_{n=0}^{N S V} \sum_{j=1}^{4} \frac{\mathrm{e}^{-\mathrm{i}(\omega / \alpha) r_{i j k}}}{r_{i j k}}\right\}\right\} \\
& +A \sum_{l=0}^{N S Z}\left\{\sum _ { k = 1 } ^ { 4 } \left\{\frac{\mathrm{e}^{-\mathrm{i}(\omega / \alpha) r_{00 k}}}{r_{00 k}}+\sum_{n=0}^{N S V} \sum_{j=1}^{4} \frac{\mathrm{e}^{-\mathrm{i}(\omega / \alpha) r_{0 j k}}}{r_{0 j k}}\right.\right. \\
& \left.+\sum_{m=1}^{N S H} \sum_{i=1}^{4}\left\{\frac{\mathrm{e}^{-\mathrm{i}(\omega / \alpha) r_{i 0 k}}}{r_{i 0 k}}+\sum_{n=0}^{N S V}\left[\sum_{j=1}^{4} \frac{\mathrm{e}^{-\mathrm{i}(\omega / \alpha) r_{i j k}}}{r_{i j k}}\right]\right\}\right\},
\end{aligned}
$$

where NSZ represents the number of sources used in the $z$ direction that are needed for the correct definition of the acoustic signal.

\subsection{GREEN FUNCTIONS FOR TWO-AND-A-HALF DIMENSIONAL PROBLEMS}

(a) Horizontal barrier in position $y=0$,

$$
\hat{p}\left(\omega, x, y, k_{z}\right)=B \sum_{j=0}^{1} \mathrm{H}_{0}\left(k_{\alpha} r_{0 j}\right) \quad(n=0)
$$

with $B=-\mathrm{i} A / 2 . \underline{z}_{k}$ and $r_{i j}=\sqrt{\left(\underline{x}_{i}\right)^{2}+\left(\underline{y}_{j}\right)^{2}}$ are defined in Appendix A. 
(b) Corner created by a vertical barrier placed on $x=d_{1}$ and another, horizontal, on $y=0$ :

$$
\hat{p}\left(\omega, x, y, k_{z}\right)=B \sum_{i=0}^{1} \sum_{j=0}^{1} \mathrm{H}_{0}\left(k_{\alpha} r_{i j}\right) \quad(n=0, m=-1) .
$$

(c) Layer bounded by horizontal barriers at distance $y=0$ and $d_{2}$ :

$$
\hat{p}\left(\omega, x, y, k_{z}\right)=B\left[\mathrm{H}_{0}\left(k_{\alpha} r_{00}\right)\right]+B\left\{\sum_{n=0}^{N S V}\left[\sum_{j=1}^{4} \mathrm{H}_{0}\left(k_{\alpha} r_{0 j}\right)\right]\right\} .
$$

(d) Layer bounded by horizontal barriers at distance $y=0$ and $d_{2}$ and another, vertical, at $x=d_{1}$.

$$
\hat{p}\left(\omega, x, y, k_{z}\right)=B \sum_{i=0}^{1}\left\{\left[\mathrm{H}_{0}\left(k_{\alpha} r_{i 0}\right)\right]+\left\{\sum_{n=0}^{N S V}\left[\sum_{j=1}^{4} \mathrm{H}_{0}\left(k_{\alpha} r_{i j}\right)\right]\right\}\right\} \quad(m=-1) .
$$

(e) Tunnel created by horizontal barriers at distance $y=0$ and $d_{2}$ and vertical ones placed at $x=0$ and $d_{1}$ :

$$
\begin{aligned}
\hat{p}\left(\omega, x, y, k_{z}\right)= & B\left\{\left[\mathrm{H}_{0}\left(k_{\alpha} r_{00}\right)\right]+\left\{\sum_{n=0}^{N S V}\left[\sum_{j=1}^{4} \mathrm{H}_{0}\left(k_{\alpha} r_{0 j}\right)\right]\right\}\right\} \\
& +B\left\{\sum_{m=1}^{N S H}\left\{\sum_{i=1}^{4}\left\{\left[\mathrm{H}_{0}\left(k_{\alpha} r_{i 0}\right)\right]+\left\{\sum_{n=0}^{N S V}\left[\sum_{j=1}^{4} \mathrm{H}_{0}\left(k_{\alpha} r_{i j}\right)\right]\right\}\right\}\right\} .\right.
\end{aligned}
$$

(f) Space bounded by a vertical barrier at distance $z=d_{3}$ (see Figure $1(\mathrm{~h})$ ),

$$
p(\omega, x, y, z)=\frac{2 \pi}{L} \sum_{m=-M}^{m=M} \hat{p}\left(\omega, x, y, k_{z m}\right) E_{1} \quad(l=-1),
$$

in which $E_{1}=\mathrm{e}^{\mathrm{i} k_{z m}\left(\underline{z}_{0}\right)}+\mathrm{e}^{\mathrm{i} k_{z m}\left(\underline{z}_{1}\right)}$.

(g) Layer bounded by two barriers placed at $z=0$ and $d_{3}$ (see Figure 1(i)),

$$
p(\omega, x, y, z)=\frac{2 \pi}{L} \sum_{m=-M}^{m=M} \hat{p}\left(\omega, x, y, k_{z m}\right) E_{2}
$$

in which $E_{2}=\mathrm{e}^{\mathrm{i} k_{z m}\left(z-z_{0}\right)}+\sum_{l=0}^{N S Z}\left[\sum_{k=1}^{4} \mathrm{e}^{\mathrm{i} k_{z m} z_{k}}\right]$.

\section{SOUND ENERGY ABSORPTION}

If the barriers are not perfectly reflective, only a fraction of the incident sound energy will be reflected from the wall. This energy can be accounted for by its absorption coefficient $\alpha$, that is, the fraction of the incident sound energy absorbed by the wall. In the models given above, reflection coefficients $(\sqrt{1-\alpha})$ can be introduced in the response each time the pulse produced by the virtual sources strikes barriers that are not perfectly reflective. The frequency-dependent absorption coefficients can thus also be contemplated. Furthermore, this process allows the simultaneous use of different absorption coefficients $(\alpha)$ on each wall. 
It is important to note that this formulation does not take into account diffracted waves that may travel as guided waves along the walls.

\section{SIGNALS IN THE TIME DOMAIN}

The pressure field in the space and time domains are obtained by using the Fourier numerical tranforms in both $k_{z}$ and in the frequency domain. The dynamic excitation is idealized as an incident pulse with the time variation of a Ricker wavelet (equation (18)). This wavelet is chosen because it decays rapidly in both time and frequency, reducing computational effort and allowing easier interpretation of the time series (sequence of relatively narrow pulses).

The time dependence of the input signal is defined as

$$
u(\tau)=A\left(1-2 \tau^{2}\right) \mathrm{e}^{-\tau^{2}}
$$

where $A$ is the amplitude, $\tau=\left(t-t_{s}\right) / t_{0}, t$ refers to time, $t_{s}$ is the time at which the maximum occurs, while $\pi t_{0}$ is the characteristic period of the wavelet. Its Fourier transform is given by

$$
U(\omega)=A\left[2 \sqrt{\pi} t_{0} \mathrm{e}^{-\mathrm{i} \omega t_{s}}\right] \Omega^{2} \mathrm{e}^{-\Omega^{2}},
$$

in which $\Omega=\omega t_{0} / 2$.

\section{APPLICATIONS}

Two models are used to illustrate the importance of the Green functions presented in this paper. In the first, the 3-D Green functions are used to calculate the pressure field inside a rectangular parallelepiped room. This model ascribed frequency-independent absorption capacities to the wall, ceiling and floor surfaces. The second model evaluates the acoustic scattering by an infinitely long rigid barrier in the vicinity of a tall building subjected to a point pressure load, using the BEM, where the analytical solution of a spatially sinusoidal line source is used. Both the ground and the building surfaces were considered to be non-absorbing.

\subsection{CALCULATION OF THE PRESSURE FIELD INSIDE A RECTANGULAR PARALLELEPIPED ROOM}

The Green function in equation (10) is used to calculate the 3-D pressure wavefield generated inside a closed rectangular room, with dimensions $d_{1}=20.0 \mathrm{~m}, d_{2}=15.0 \mathrm{~m}$, and $d_{3}=40.0 \mathrm{~m}$ (see Figure 3) illuminated by a point pressure load placed at position $\left(x_{0}=10.0 \mathrm{~m} ; y_{0}=2.0 \mathrm{~m}, z_{0}=2.0 \mathrm{~m}\right)$, which produces sound waves propagating with a velocity of $340 \mathrm{~m} / \mathrm{s}$. Three situations were analyzed, in which the walls, ceiling and floor were ascribed different absorption capacities (see Table 1).

Time responses were calculated for the receiver placed as shown in Figure $3(x=10 \cdot 0 \mathrm{~m}$; $y=1.5 \mathrm{~m} ; z=38.0 \mathrm{~m})$. The calculations were made in the frequency domain $[1.5$, $12288 \mathrm{~Hz}$ ], with a frequency increment of $1.5 \mathrm{~Hz}$. This allowed the time-domain analysis to be as long as $T=666.6 \mathrm{~ms}$. The signals in time were then calculated by applying an inverse Fourier transform, using a Ricker pulse with a characteristic frequency of $4000 \mathrm{~Hz}$.

Figure 4 displays the response recorded at the receiver. Each signal in time consisted of a set of pulses that was located in time according to the reflections on the barriers. The effect 

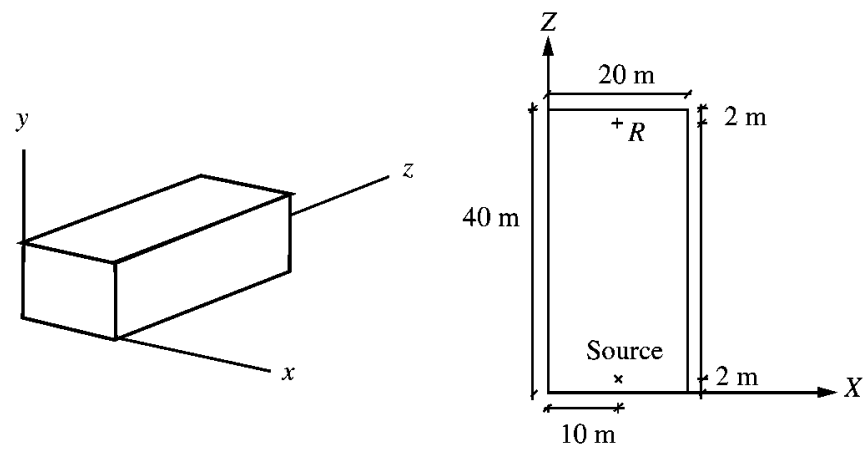

Figure 3. Models studied. Receiver position.

TABLE 1

Absorption coefficients

\begin{tabular}{lcccccc}
\hline & Ceiling & Floor & Rear wall & Front wall & Right wall & Left wall \\
\hline Case 1 & $0 \cdot 0$ & $0 \cdot 19$ & $0 \cdot 0$ & $0 \cdot 0$ & $0 \cdot 0$ & $0 \cdot 0$ \\
Case 2 & $0 \cdot 0$ & $0 \cdot 19$ & $0 \cdot 75$ & $0 \cdot 0$ & $0 \cdot 0$ & $0 \cdot 0$ \\
Case 3 & $0 \cdot 75$ & $0 \cdot 19$ & $0 \cdot 75$ & $0 \cdot 0$ & $0 \cdot 0$ & $0 \cdot 0$ \\
\hline
\end{tabular}

of the floor's absorption (case 1) is observed as a slight but gradual decrease in the amplitude of the response as the time increases. The introduction of absorption in the rear wall (case 2) produces a drop in amplitude that is somewhat faster than that seen in the preceding case, obviously due to the presence of the additional absorbing element. In case 3 , the responses have a very distinctive behaviour. The response now clearly decreases much faster with time, with fewer significant pulses. The high absorption coefficient assumed for the ceiling and rear wall is responsible for this change. It is interesting to see that some pulses still arrive at later times, signifying that they are reflections from the lateral walls, while reflections from the ceiling and rear wall have now almost disappeared.

The responses show that, after the first arrivals, the signal is followed by a set of pulses with progressively lower amplitude, as a result of absorption at the barriers. To better illustrate this fall in amplitude, the energy responses were calculated by using the expression $10 \log \left(p^{2} /\left(2 \times 10^{-5}\right)^{2}\right)$, also illustrated in Figure 4 .

The results indicate that a set of small pulses, not visible in the time domain, exist prior to the beginning of the real dynamic response. This phenomenon corresponds to the wrap-around response beyond $T=1 / \Delta f$, which even the use of complex frequencies $\left(\omega_{c}=\omega-\mathrm{i} \eta\right)$ could not completely prevent, given both the high reverberation time involved and the high value of $\Delta f$. Note that as the reverberation time decreases from cases 1 to 3 , the amplitude of this set of pulses also decreases.

\subsection{SCATTERING OF 3-D SOUND SOURCES BY RIGID BARRIERS IN THE VICINITY OF A TALL BUILDING}

The scattering of a 2-D acoustic medium when illuminated by a pressure point load (known as a 2-1/2-D problem), can be solved as a discrete summation of the 2-D BEM 
Case 1

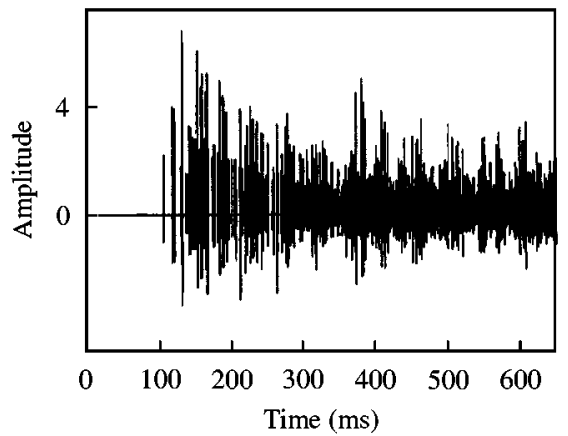

Case 2

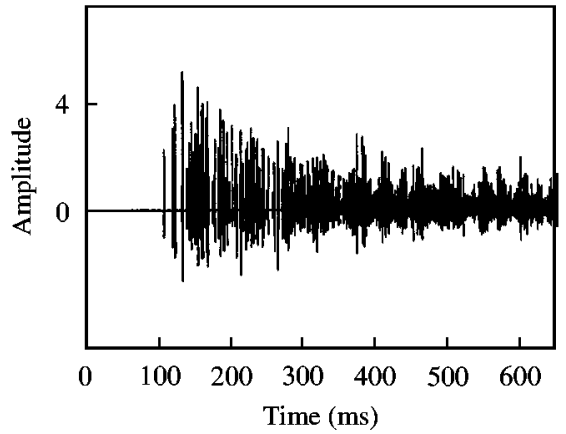

Case 3

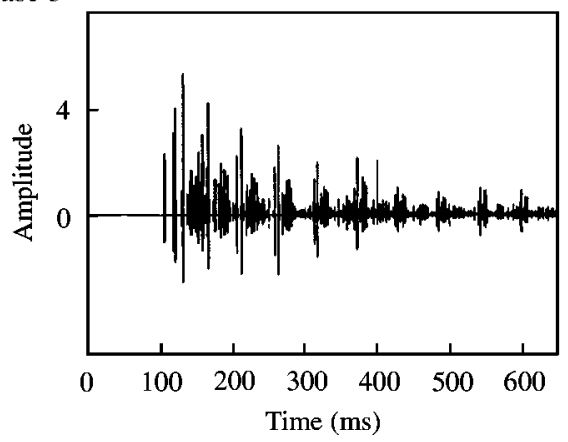

$10 \log \left(p^{2} /\left(2 \times 10^{-5}\right)^{2}\right)$
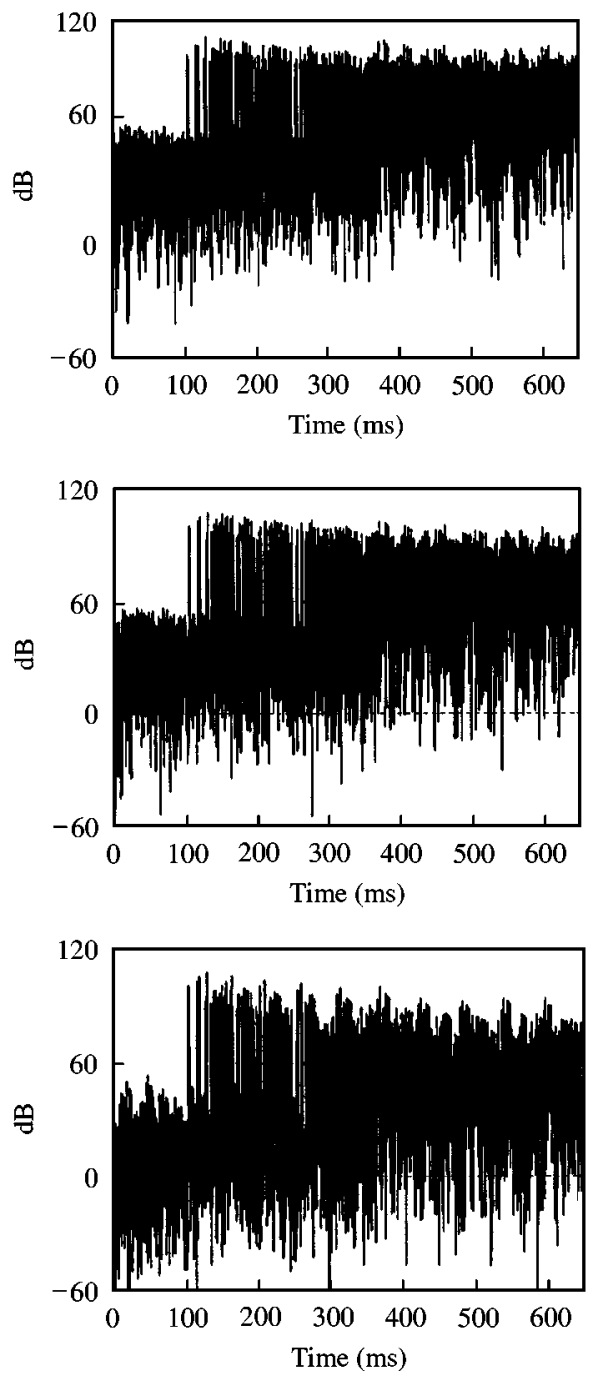

Figure 4. Calculated responses.

solutions for different $k_{z}$ wavenumbers. This is achieved by assuming the existence of an infinite number of virtual point sources, spaced at equal intervals along the $z$-axis, with the distance between them being large enough to prevent the response from suffering spatial contamination [16]. The 3-D field is subsequently obtained with the inverse spatial Fourier transform technique.

We do not give full details of the BEM formulation applied to the type of scattering problem that is described here, since these can be easily found in the literature (e.g. see the book by Manolis and Beskos [17]), but it should be noted that the following integral has to be solved for each 2-D problem:

$$
\mathrm{H}^{k l}=\int_{C_{l}} \mathrm{H}\left(\underline{x}_{k}, \underline{x}_{l}, n_{l}\right) \mathrm{d} C_{l},
$$


where $\mathrm{H}^{k l}$ is the pressure velocity component at $\underline{x}_{k}$ resulting from pressure load $x_{l}$, and $n_{l}$ is the unit outward normal for the $l$ th boundary segment $C_{l}$. The pressure velocity function is obtained by differentiating equation (12) in relation to the unit outward normal.

Equation (20) is applied to the set of constant boundary elements used to discretize the acoustic barrier boundary to give a system of equations that relates the pressure field to a set of nodal pressure values. As the interpolation functions used in the present BEM are constant, the nodal points are identified as the median points of the boundary segments. The nodal pressure values are calculated once the equations have been mathematically manipulated and zero velocity applied to the outward normal direction of the boundary elements. Gauss-Legendre quadrature, with six integration points, is used for the integrations in equation (20), and, as the acoustic barrier is taken to have a certain thickness, higher order Gauss-Legendre quadrature is used for the numerical integration when the distance from the loaded element to the element being integrated is small.

The nodal pressure values are then employed to calculate the scattered pressure field, at any point within the medium:

$$
p_{s c a}^{k}=\sum_{l=1}^{N} p^{l} \mathrm{H}^{k l} .
$$

Here, $p_{s c a}^{k}$ represents the scattered pressure field at receiver $k, N$ is the total number of boundary elements, and $p^{l}$ the nodal pressure value at element $l$.

The method described above has been used to study the influence of an acoustic barrier placed between a point sound pressure load and a tall building. The ground and the building were taken to be non-absorbing and the acoustic medium to have a pressure wave velocity of $340 \mathrm{~m} / \mathrm{s}$. A harmonic point source is positioned $0.6 \mathrm{~m}$ above the surface of the ground and at a distance of $25.0 \mathrm{~m}$ from the building. A $6.0 \mathrm{~m}$ acoustic barrier is placed $20.0 \mathrm{~m}$ from the building, with the aim of reducing the sound at its façade. Figure 5 gives the layout of the problem. In this figure, the dots placed along the vertical plane, $x=0 \cdot 0$, represent the façade of the tall building.

The barrier is $0.2 \mathrm{~m}$ thick and has been discretized with boundary elements, the number of which was determined by the ratio between the wavelength and the length of the boundary elements, fixed at 8 . The minimum number of boundary elements is 32 .

The response for the first simulation was captured by a grid of receivers arranged on a vertical plane $0.5 \mathrm{~m}$ from the rigid wall. The receivers were spaced at intervals 0 1.0 m

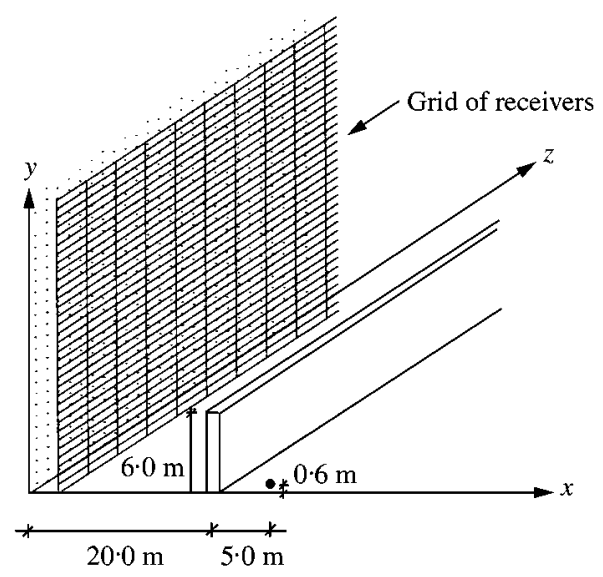

Figure 5. Geometry of the problem. 

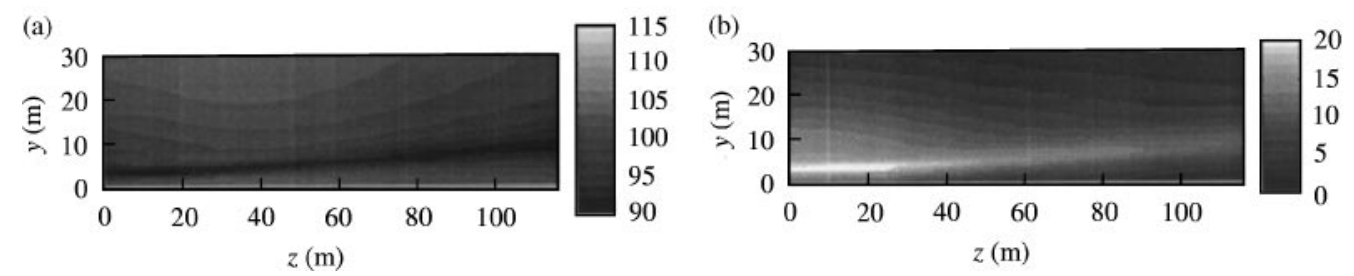

Figure 6. Sound pressure along a vertical plane $0.5 \mathrm{~m}$ away from the building: (a) pressure level; (b) pressure attenuation.
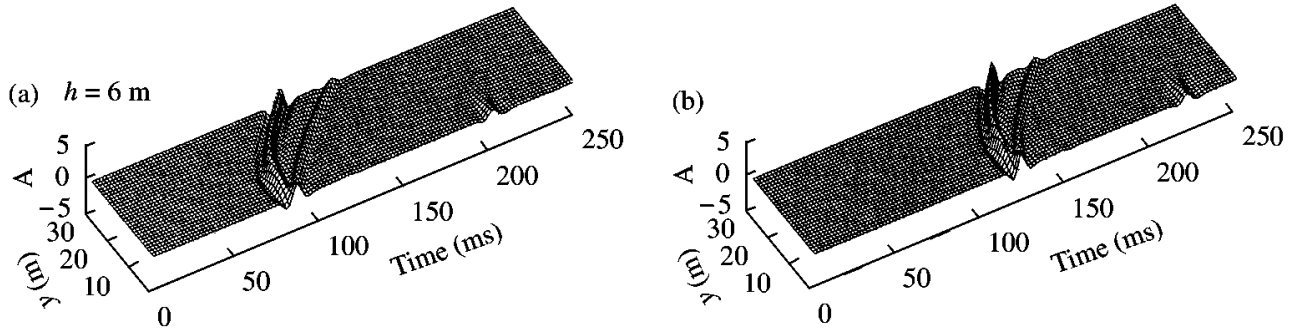

Figure 7. Time response at receivers $0.5 \mathrm{~m}$ away from the building: (a) $z=0.0 \mathrm{~m}$; (b) $z=30 \cdot 0 \mathrm{~m}$.

vertically and $4.0 \mathrm{~m}$ longitudinally. Calculations were performed for a frequency range of $2-256 \mathrm{~Hz}$, with increments of $2 \mathrm{~Hz}$, giving $0.5 \mathrm{~s}$ as the total time frame for the response. A Ricker wavelet, with frequency of $100 \mathrm{~Hz}$, was taken as the source time dependence.

The sound pressure level and its attenuation, once the barrier is placed between the source and the building, are given in Figures 6(a) and 6(b). It can be seen from these figures that the performance of the barrier is poorer close to the ground, owing to the interaction of the direct field, diffracted by the barrier, and the field reflected by the ground. The receivers placed higher up, however, show improving efficiency, with a maximum being achieved at a height of around $4 \mathrm{~m}$ for $z=0.0 \mathrm{~m}$. After this, the efficiency of the barrier drops to the point where the performance is poorer than in a situation where there is no barrier. The performance is not constant along the $z$-axis. A plot of the points of maximum efficiency for consecutive vertical $z$ planes is a sloping line, which indicates better performances for the receivers placed higher up, as $z$ increases. The reflections on the ground near the building also appear to gain in importance as $z$ increases.

For a better appreciation of the way the sound propagates from its source to the receivers, the time responses for receivers placed at $z=0.0 \mathrm{~m}$ and $30.0 \mathrm{~m}, 0.5 \mathrm{~m}$ distant from the building, are given.

The time responses at receivers placed at $z=0.0 \mathrm{~m}$, the source plane, are given in Figure 7(a). They represent the effects of the reflection of a series of incident pulses and the result of their reflections on the ground, wall and barrier. The arrival times of the different pulses agree with those calculated using the acoustic ray theory. A second pulse is recorded just after the first one, and the receivers placed higher up show this more clearly. The second pulse results from a prior reflection on the ground.

After more time has elapsed, a third pulse is recorded. This is caused by the energy occurring between the barrier and the building, which gives rise to secondary reverberations. The interaction of the barrier and the wall creates additional pulses, but these are not visible in the time frame used. The approximate difference in time separating 
these pulses is represented by $40.0 \mathrm{~m} / 340 \cdot 0 \mathrm{~ms}=117.6 \mathrm{~ms}$. Their amplitude decreases as the energy dissipates.

The responses for $z=30.0 \mathrm{~m}$ at the same set of receivers are shown in Figure 7(b). The pulses arrive later because their trajectories are longer, although their characteristics are generally similar to those found for $z=0.0 \mathrm{~m}$. Comparisons of the two sets of responses show a very small drop in amplitude, as a result of the addition of the directly incident pulses to the pulses reflected on to the building.

Notice that, the façade of the building was modelled as a virtual infinite plane. However, the results obtained are still valid when a tall building is analyzed. Indeed, the scattered field, generated for waves illuminating the virtual plane above the top level of the building, is small. In addition, the larger amount of energy reflected on these points is scattered to higher levels, while much less energy is reflected to lower levels.

\section{CONCLUSIONS}

The analytical equations used to solve problems of acoustic wave scattering are presented for situations where the scattering is caused by point and line acoustic sources, placed either within an unbounded medium or in confined spaces. The amplitude of such acoustic sources may vary in one direction. The equations use complex frequencies and are formulated in the frequency domain. Inverse Fourier transforms are used to get the temporal responses.

Pressure fields inside a rectangular parallelepiped space, and near a sound barrier, were determined in the frequency and time domains to demonstrate that the equations are applicable to these two situations. The results corresponded to what was expected theoretically, thus indicating that the technique could be used to model acoustic pressure inside both open and closed spaces, making it possible to use absorption coefficients defined as function of the frequency.

\section{REFERENCES}

1. J. B. Allen and D. A. Berkley 1979 Journal of Acoustical Society of America 65, 943-950. Image method for efficiently simulating small room acoustics.

2. R. N. S. Hammad 1988 Applied Acoustics 24, 211-228. Simulation of noise distribution in rectangular rooms by means of computer modeling techniques.

3. H. LEE and B.-H. LEE 1988 Applied Acoustics 24, 87-115. An efficient algorithm for the image model technique.

4. A. Kulowski 1982 Applied Acoustics 15, 263-274. Error investigation for the ray tracing technique.

5. A. KulOWSKI 1985 Applied Acoustics 18, 449-469. Algorithmic representation of the ray tracing technique.

6. Y. W. LAm 1994 Applied Acoustics 42, 29-40. Using Maekawa's chart to calculate finite length barrier insertion loss.

7. A. Muradali and K. R. Fyfe 1998 Applied Acoustics 53, 49-75. A study of 2D and 3D barrier insertion loss using improved diffraction-based methods.

8. L. A. Lacerda, L. C. Wrobel and W. J. Mansur 1997 Journal of Sound and Vibration 202, 235-347. A dual boundary element formulation for sound propagation around barriers over an infinite plane.

9. L. A. Lacerda, L. C. Wrobel, H. Power and W. J. Mansur 1998 Journal of Acoustical Society of America. 104, 671-678. A novel boundary integral formulation for three-dimensional analysis of thin acoustic barriers over an impedance plane.

10. L. ZHANG and A. K. CHOPRA 1991 International Journal of Earthquake Engineering and Structural Dynamics 20,911-926. Three dimensional analyses of spatially varying ground motions around a uniform canyon in a homogeneous half-space. 
11. A. A. Stamos and D. E. Beskos 1996 Journal of Soil Dynamics and Earthquake Engineering 15, 111-118. 3-D seismic response analysis of long lined tunnels in half-space.

12. A. TADEU and L. Godinho 1999 EABE-Engineering Analysis with Boundary Elements 23, 745-755. 3D wave scattering by a fixed cylindrical inclusion submerged in a fluid medium.

13. A. J. B. TADEU and J. M. P. ANTÓNIO 2000 EABE-Engineering Analysis with Boundary Elements 24, 131-144. Use of constant, linear and quadratic boundary elements in $3 \mathrm{D}$ wave diffraction analysis.

14. P. Jean, J. Defrance and Y. Gabillet 1999 Journal of Sound and Vibration 226, 201-216. The importance of source type on the assessment of noise barriers.

15. E. KAUSEL and J. M. ROESSET 1992 Journal of Engineering Mechanics American Society of Civil Engineers 118, 721-734. Frequency domain analysis of undamped systems.

16. M. Bouchon and K. AKi 1977 Bulletin of Seismic Society of America 67, 259-277. Discrete wave-number representation of seismic-source wave field.

17. G. D. MANOLIS and D. E. Beskos 1988 Boundary Element Methods in Elastodynamics. London: Unwin Hyman (sold to Chapman \& Hall).

APPENDIX A: DEFINITIONS OF $\underline{x}_{i}, \underline{y}_{j}, \underline{z}_{k}, r_{i j k}$ AND $r_{i j}$

$r_{i j k}=\sqrt{\left(\underline{x}_{i}\right)^{2}+\left(\underline{y}_{j}\right)^{2}+\left(\underline{z}_{k}\right)^{2}}$ and $r_{i j}=\sqrt{\left(\underline{x}_{i}\right)^{2}+\left(\underline{y}_{j}\right)^{2}}$, used throughout the text, to be calculated.

$$
\begin{array}{cccc}
\underline{x}_{0}=\left(x-x_{0}\right), & \underline{y}_{0}=\left(y-y_{0}\right), & \underline{z}_{0}=\left(z-z_{0}\right), \\
\underline{x}_{1}=\left(x+x_{0}+2 d_{1} m\right), & \underline{y}_{1}=\left(y+y_{0}+2 d_{2} n\right), & \underline{z}_{1}=\left(z+z_{0}+2 d_{3} l\right), \\
\underline{x}_{2}=\left(x-2 d_{1}-x_{0}-2 d_{1} m\right), & \underline{y}_{2}=\left(y-2 d_{2}-y_{0}-2 d_{2} n\right), & \underline{z}_{2}=\left(z-2 d_{3}-z_{0}-2 d_{3} l\right), \\
\underline{x}_{3}=\left(x+2 d_{1}-x_{0}+2 d_{1} m\right), & \underline{y}_{3}=\left(y+2 d_{2}-y_{0}+2 d_{2} n\right), & \underline{z}_{3}=\left(z+2 d_{3}-z_{0}+2 d_{3} l\right), \\
\underline{x}_{4}=\left(x-2 d_{1}+x_{0}-2 d_{1} m\right), & \underline{y}_{4}=\left(y-2 d_{2}+y_{0}-2 d_{2} n\right), & \underline{z}_{4}=\left(z-2 d_{3}+z_{0}-2 d_{3} l\right) .
\end{array}
$$

